\title{
A New Standard Formula for Seasonal Growth of Fish in Population Dynamics ${ }^{* 1}$
}

\author{
Tetsuro Akamine*2 \\ (Received May 20, 1993)
}

\begin{abstract}
This formula is based on the Richards formula, which includes well-known growth formulae, and is invariant for allometry. It is expanded by exchanging the independent variable from time to its function, in other words the cumulative water temperature. This standard formula includes the method of substituting values for the cumulative water temperature into the time term, the mathematical model of adding sine functions to the time term, and switched growth rate models. This standard model is given by general theory, which makes the character of parameters clear and helps to analyze the growth formula statistically.
\end{abstract}

Many formulae have been proposed for fish growth. In particular, the four well-known formulae (von Bertalanffy, its cubic, Gompertz, and longistic) are widely used. Richards ${ }^{1}$ original formula, which includes all of these four formulae, is not easy to understand nor is it widely used. It has been known since the 1960's that substituting the value of cumulative water temperature (day degrees) into the time term of the von Bertalanffy formula is useful for describing fish growth. ${ }^{23}$ On the other hand, the von Bertalanffy formulae, developed by adding a sine function to its time term, have been studied mathematically since the 1970 's. $^{8}$ '

I have studied these formulae mathematically, ${ }^{4,3)}$ and have obtained a useful standard formula. This formula makes it easy to understand the Richards formula, and to indicate the method of substituting the value for the cumulative water temperature is identical to the model of adding a sine function. In this paper, this new standard formula is explained theoretically.

\section{General Growth Formula}

\section{Traditional Growth Formula}

Many growth formulae are given as the solutions to differential equations. These are expressed generally by the following differential equation:

$$
\frac{\mathrm{d} w}{\mathrm{~d} t}=g(w)
$$

It gives

$$
\frac{\mathrm{d} w}{g(w)}=\mathrm{d} t .
$$

Integrating both sides of this equation gives

$$
G(w)=t+c,
$$

where $c$ is the integration constant, and $G(w)$ is given by

$$
G(w)=\int \frac{\mathrm{d} w}{g(w)} .
$$

Therefore, the general solution of Eq. (3) is

$$
w=G^{-1}(t+c),
$$

where $G^{-1}$ denotes the inverse function of $G$. This is the general formula including the above four well-known formulae.

In mathematics, the differential equation (1) and its integral solution (5) are identical. However, the latter is more useful than the former in practice. The four well-known formulae mentioned above are,widely used for two reasons: one is that their formulae are based on differential equations, and the other is that their functions $G^{-1}$ are simple and easy to use.

\section{Expansion by Periodic Function}

Equation (1) is not enough to describe fish growth in detail. The best differential equation may be

$$
\frac{\mathrm{d} w}{\mathrm{~d} t}=h(w, t) \text {. }
$$

where $w$ is the body weight or length, and $t$ is time. However, with this equation it is difficult to ob-

*1 Contribution B No. 81 from the National Research Institute of Fisheries Science.

*2 National Research Institute of Fisheries Science, Fukuura, Kanazawa, Yokohama 236, Japan (赤 嶺達郎: 中央水産研究所)。 
tain an integral formula in general. Instead of Eq. (6), we consider the following equation:

$$
\frac{\mathrm{d} w}{\mathrm{~d} t}=g(w) f(t) .
$$

This is a "separation of variables" type equation, and we can solve this equation as follows: Let

$$
T=F(t)=\int f(t) \mathrm{d} t .
$$

Differentiating both sides of this equation gives

$$
\mathrm{d} T=f(t) \mathrm{d} t .
$$

Substituting Eq. (9) into Eq. (7) gives

$$
\frac{\mathrm{d} w}{g(w)}=f(t) \mathrm{d} t=\mathrm{d} T .
$$

In the same way from Eq. (2) to Eq. (5), the general solution of Eq. (10) is

$$
w=G^{-1}(T+c)=G^{-1}(F(t)+c) .
$$

This is an expanded growth formula.

If $f(t)$ is defined to be the water temperature, $\left.{ }^{4}\right)$ $F(t)$ refers to the cumulative water temperature. Therefore, we get a mathematical model (11) for the method of substituting the value for the cumulative water temperature into the time term of growth formula.

Although we can use any type of $f(t)$, the most important type in biology (and mathematics) is a periodic function:

$$
f(t+1)=f(t),
$$

where time units are measured in years. For this periodic function, the following standardization:

$$
\int_{0}^{1} f(t) \mathrm{d} t=F(1)-F(0)=1
$$

is necessary to compare growth formulae, ${ }^{(2)}$ and in particular the values of the growth coefficient $K$ (see the next section).

\section{New Standard Formula}

Richards differential equation is given by Eq. (1) when $g(w)$ is assumed to be

$$
g(w)=K w^{1-\left(w / w_{\infty}\right)^{r}},
$$

where $w_{\infty}, K$, and $r$ mean the asymptotic body weight or length, the growth coefficient, and the decision factor of the curve shape, respectively. This function gives

$$
G(w)=-\frac{1}{K} \ln \frac{\left(w_{\infty} / w\right)^{r}-1}{r} .
$$

Therefore, we obtain the following general solution:

$$
w=\frac{w_{\infty}}{\left\{1+r \mathrm{e}^{-\boldsymbol{K}(t+c)}\right\}^{1 / r}} .
$$

This is the general formula of the Richards equation. The range of parameter $r$ is $-\infty \leqq r \leqq \infty$. In fisheries population dynamics, it is usually called the generalized von Bertalanffy growth formula. ${ }^{7,8)}$

It is well known that Eqs. (14) and (16) are identical to von Bertalanffy, its cubic, Gompertz, and logistic formulae when $r=-1,-1 / 3,0$, and 1 , respectively. The Gompertz formula is easily derived by using the following definitions of exponent and logarithm:

$$
\begin{aligned}
& y=\mathrm{e}^{x}=\lim _{r \rightarrow 0}(1+r x)^{1 / r}, \\
& x=\ln y=\lim _{r \rightarrow 0} \frac{y^{r}-1}{r} .
\end{aligned}
$$

The general formula (16) has an integration constant $c$. For practical use, we must decide the value of $c$ to get a particular solution of the differential equation. In fisheries population $d y$ namics, we use fhe following value:

$$
c=-t_{0} \text {. }
$$

Integration makes one parameter $t_{0}$. This is ob. tained by the following initial condition:

$$
(t, w)=\left(t_{0}, G^{-1}(0)\right),
$$

where Eq. (15) gives

$$
G^{-1}(0)=\frac{w_{\infty}}{(1+r)^{1 / r}} .
$$

This is the inflection point when $r>-1$. The graph of Eq. (16) combined with Eq. (17) is shown in Fig. 1 for various values of parameter $r$.

From Eqs. (11) and (16), we decide the new standard formula expanded by using a periodic function to be

$$
W=c \begin{gathered}
w_{\infty} \\
{\left[1+r \mathrm{e}^{-K\left(F(\ell)-F\left(t_{0}\right) \mid\right.}\right]^{1 / r}}
\end{gathered},
$$

where $F(t)$ is defined by Eqs. (8), (12), and (13). In this formula, the initial condition (18) gives the following integral constant:

$$
c=-F\left(t_{0}\right)
$$

instead of Eq. (17)." Although another formula 


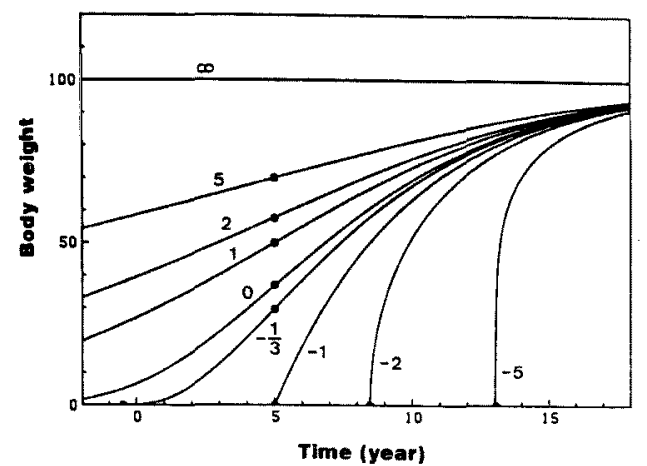

Fig. 1. Graph of Richards formula (16) combined with $\mathrm{Eq} .(17)$ in the text.

Parameters are $w_{\infty}=100, K=0.2, t_{0}=5, r=$ $-5,-2,-1,-1 / 3,0,1,2,5, \infty$. Solid circles are the influection points.

is popular in agricultural science, ${ }^{9)}$ it is not traditional in fisheries population dynamics (see Appendex A).

\section{Some Models}

\section{Cumulative Water Temperature Model}

This model has been used for mussels. ${ }^{10,11)}$ It is better to standardize temperature by Eq. (13). Thus we can compare values of the parameter $K$ with the other mathematical models mentioned later.

\section{Sine Function Model}

This model is defined as follows:

$$
\begin{aligned}
f(t) & =1+A \cos 2 \pi\left(t-t_{1}\right), \\
F(t) & =t+\frac{A}{2 \pi} \sin 2 \pi\left(t-t_{1}\right),
\end{aligned}
$$

where $A \geqq 0$ and $0 \leqq t_{1}<1$. Parameter $A$ refers to the amplitude of oscillation, and $t_{1}$ signifies the maximum point of $f(t)$. When $A>1$, growth rate is temporally negative. Eq. (23) gives

$$
t-\frac{A}{2 \pi} \leqq F(t) \leqq t+\frac{A}{2 \pi} .
$$

Therefore, the graph of this model is osculated by two original Richards formulae (Fig. 2).,

\section{Switched Model}

Pitcher and MacDonald ${ }^{12)}$ presented two models: one was the sine wave growth model mentioned above, while the other was this switched growth model. Akamine ${ }^{13,14}$ rewrote the latter mathematically, which is standardized by Eq. (13) as

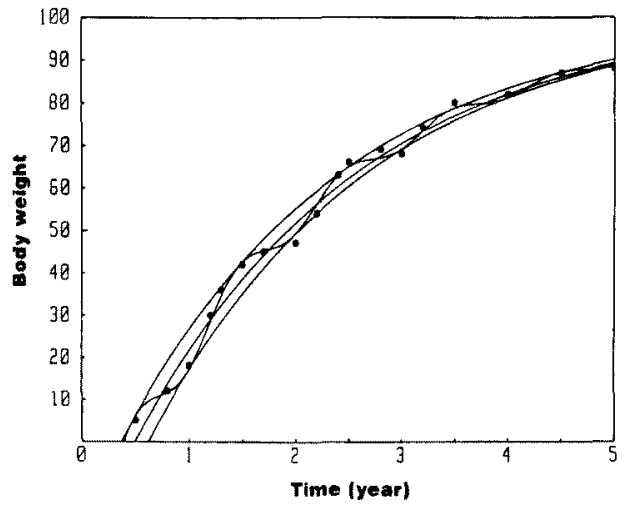

Fig. 2. Graph of von Bertalanffy formula combined with the sine function model (23) in the text.

The original and the two osculating formulae are also shown. Solid circles are data to be fitted.

follows:

$$
f(t)= \begin{cases}\frac{1}{1-T}, & \left(0 \leqq t<t_{2}, t_{3} \leqq t<1\right) \\ 0, & \left(t_{\mathrm{2}} \leqq t<t_{3}\right)\end{cases}
$$

where $T=t_{3}-t_{\mathrm{a}}>0$. This gives

$$
F(t)=\left\{\begin{array}{cc}
n+\frac{t-n}{1-T}, & \left(n \leqq t<n+t_{2}\right) \\
n+\frac{t_{2}}{1-T}, & \left(n+t_{2} \leqq t<n+t_{3}\right) \\
n+\frac{t-n-T}{1-T}, & \left(n+t_{3} \leqq t<n+1\right)
\end{array}\right.
$$

where $n$ is the integral part of $t$. Although this growth model is not smooth, it has no shrinkage.

\section{Switched Sine Function Model}

The sine function models can be also switched off. For example, we consider Eq. (22) to switch off: When $A>1$, it is written as

$$
f^{*}(t)= \begin{cases}\frac{f(t)}{1-s}, & \left(f(t) \geqq 0: 0 \leqq t<t_{2}, t_{3} \leqq t<1\right) \\ 0, & \left(f(t)<0: t_{2} \leqq t<t_{3}\right)\end{cases}
$$

where $f(t)$ is Eq. (22), and

$$
\begin{aligned}
& s=\int_{t_{2}}^{t_{3}} f(t) \mathrm{d} t=F\left(t_{3}\right)-F\left(t_{2}\right)<0, \\
& f\left(t_{2}\right)=f\left(t_{3}\right)=0 .
\end{aligned}
$$

The last equation gives the values of $t_{2}$ and $t_{3}$. 
When $t_{1}<t_{2}$, it gives

$$
\begin{aligned}
& t_{2}=t_{1}+\frac{1}{2 \pi} \arccos \left(-\frac{1}{A}\right), \\
& t_{3}=1+2 t_{1}-t_{2} .
\end{aligned}
$$

This function gives

$$
F^{*}(t)= \begin{cases}n+\frac{F(t-n)}{1-s}, & \left(n \leqq t<n+t_{2}\right) \\ n+\frac{F\left(t_{2}\right)}{1-s}, & \left(n+t_{2} \leqq t<n+t_{3}\right) \\ n+\frac{F(t-n)-s}{1-s}, & \left(n+t_{3} \leqq t<n+1\right)\end{cases}
$$

where $F(t)$ is Eq. (23) and $n$ is the integral part of $t$. This model includes the original switched model when $f(t) \equiv 1$ where $s=t_{3}-t_{2}>0$,

\section{Seasonal Cessation Model}

Pauly et $a l{ }^{1 \mathrm{~B})}$ presented a new model. It is rewritten as follows: When $0 \leqq t_{1}<t_{2}<t_{3}<1$,

$$
f^{\circ}(t)= \begin{cases}1+\cos 2 \pi \frac{t-t_{1}}{1-T}, & \left(0 \leqq t<t_{2}\right) \\ 0, & \left(t_{2} \leqq t<t_{8}\right) \\ 1+\cos 2 \pi \frac{t-T-t_{1}}{1-T}, & \left(t_{3} \leqq t<1\right)\end{cases}
$$

where

$$
\begin{gathered}
T=t_{3}-t_{2}>0, \\
t_{2}=t_{1}+\frac{1-T}{2}, \quad t_{3}=t_{1}+\frac{1+T}{2}, \\
\int_{0}^{1} f^{\circ}(t) \mathrm{d} t=1-T .
\end{gathered}
$$

This gives

$F^{\circ}(t)$

$$
= \begin{cases}n(1-T)+S^{\circ}(t-n), & \left(n \leqq t<n+t_{2}\right) \\ n(1-T)+S^{\circ}\left(t_{2}\right), & \left(n+t_{2} \leqq t<n+t_{3}\right) \\ n(1-T)+S^{\circ}(t-n-T), & \left(n+t_{3} \leqq t<n+1\right)\end{cases}
$$

where

$$
S^{\circ}(t)=t+\frac{1-T}{2 \pi} \sin 2 \pi \frac{t-t_{1}}{1-T} .
$$

Although this growth model is smooth, it does not satisfy the standardization (13).

\section{Discussion}

The Richards formula is useful because it is invariant for the allometry:

$$
y=a x^{b},
$$

which is widely used for the relative growth. When variable $x$ belongs to the Richards formula $\left(r=r_{0}\right)$, variable $y$ also belongs to it $\left(r=r_{0} / b\right)$. In fisheries population dynamics, this characteristic is important because the allometry is used for the transformation of the body weight and length.

Pauly proposed "the generalized VBGF (von Bertalanffy growth formula)" in $1979 .{ }^{7}$ It is written as

$$
L=L_{\infty}\left(1-\mathrm{e}^{-K D\left(t-t_{0}\right)}\right)^{1 / D} .
$$

This formula is the same as the Richards formula, except that the range of parameter $D$ is limited to $0<D$. On the other hand, Schnute ${ }^{8>}$ proposed the following simultaneous differential equations:

$$
\begin{aligned}
& \frac{\mathrm{d} Y}{\mathrm{~d} t}=Y Z, \\
& \frac{\mathrm{d} Z}{\mathrm{~d} t}=-Z(a+b Z) .
\end{aligned}
$$

These equations are identical to the Richards differential equation in that parameters $a$ and $b$ are identical to $K$ and $-r$, respectively (see Appendix B). Schnute accurately derived the Richards formula from these equation. However, he called it the generalized von Bertalanffy formula when $b>0$. Although the Richards formula is well known, its character is not understood yet.

We can make many formulae like the Richards formula. For example, France and Thornley ${ }^{\theta}$ showed the Chanter formula:

$$
\begin{gathered}
\frac{\mathrm{d} w}{\mathrm{~d} t}=K w\left(1-\frac{w}{B}\right) \mathrm{e}^{-D t}, \\
w=\frac{w_{0} B}{w_{0}+\left(B-w_{0}\right) \exp \left\{-K\left(1-\mathrm{e}^{-D t}\right) / D\right\}} .
\end{gathered}
$$

This formula is identical to the logistic formula when $D \rightarrow \infty$, and the Gompertz formula when $B \rightarrow \infty$. However, this formula has no advantage over the Richards formula, for two reasons: one is that the range of this formula is part of the Richards formula $(r=0 \sim 1)$, and the other is that this formula needs two parameters for changing curve shape.

Akamine $^{4}$ considered another type of growth formula (type-2) based on a growth rate equation. For the von Bertalanffy formula, it is written as

$$
\frac{\mathrm{d} w}{\mathrm{~d} t}=K w_{\infty} \mathrm{e}^{-K\left(t-t_{0}\right)} f(t)
$$


where $f(t)$ is the periodic function (22). When $f(t)=1$, this equation is identical to the growth rate of the von Bertalanffy formula. This equation gives an analytical solution:

$$
w=w_{\infty}^{*}\left\{1-\frac{P(t)}{P\left(t_{0}\right)} \mathrm{e}^{-x\left(t-t_{0}\right)}\right\},
$$

where

$$
\begin{gathered}
w_{\infty}^{*}=w_{\infty} P\left(t_{0}\right), \\
P(t)=1+\frac{A K}{K^{2}+4 \pi^{2}} \\
\times\left\{K \cos 2 \pi\left(t-t_{1}\right)-2 \pi \sin 2 \pi\left(t-t_{1}\right)\right\} .
\end{gathered}
$$

This formula also expresses seasonal growth, and its parameters can be estimated by computer. However, this formula is not based on a differential equation, and gives no indication of the cumulative water temperature.

In 1973, Pitcher and MacDonald ${ }^{22}$ presented two seasonal growth models only for the von Bertalanffy formula. One is a sine function model related to the water temperature. However, they did not consider the magnitude of parameter $\boldsymbol{A}$ sufficiently. Pauly and Gaschütz developed this model to obtain the accurate magnitude of parameter $A$ by using the growth rate. ${ }^{3)}$ However, they did not consider the differential equation, and they used Eq. (17) instead of Eq. (21). Therefore, there are many mistakes for parameter $t_{0}$ in their model. For example, " $t_{0}$ is time when $w=0$ " and " $\sin 2 \pi\left(t_{0}-t_{1}\right)=0$ at $t=$ $t_{0}{ }^{\prime} \cdot{ }^{10)}$

Pawlak and Hanumara ${ }^{17)}$ said that Hoenig and Hanumara presented the same formula as the standard formula (20) combined with the sine function model (23) only for the von Bertalanffy formula in 1982. Although they compared between the Hoenig and Hanumara model and that of Pauly and Gashütz, they did not show that the former was better than the latter statistically. Somers ${ }^{18}$ ) obtained the same formula as Hoenig and Hanumara to correct the mistake in parameter $t_{0}$. He also considered the same differential equation as Eq. (7). Recently, Pauly et $a .^{153}$ have also adopted this formula. It is written as

$$
w=w_{\infty}\left[1-\mathrm{e}^{-\left(K\left(t-t_{0}\right)+s(t)-s\left(t_{0}\right)\right)}\right],
$$

where

$$
S(t)=\frac{A K}{2 \pi} \sin 2 \pi\left(t-t_{1}\right)
$$

However, this expression is difficult to connect with the idea of the cumulative water temperature.

On the other hand, Akamine ${ }^{4, v}$ used the following functions (type-1) for the sine function model:

$$
\begin{aligned}
& f(t)=\frac{1+a}{2}+\frac{1-a}{2} \cos 2 \pi\left(t-t_{1}\right), \\
& F(t)=\frac{1+a}{2} t+\frac{1-a}{4 \pi} \sin 2 \pi\left(t-t_{1}\right),
\end{aligned}
$$

where $-1 \leqq a \leqq f(t) \leqq 1$ and $0 \leqq t_{1}<1$. Parameter $t_{1}$ is identical to Eqs. (22) and (23). Parameter $a$ means the ratio of minimum $K$ (growth coefficient) to maximum. When $a<0$, growth rate is temporally negative. However, these equations do not satisfy standardization (13). The following equations transform Eqs. (31) and (32) to Eqs. (22) and (23), respectively.

$$
\begin{aligned}
& K \leftarrow \frac{1+a}{2} K, \\
& A=\frac{2}{1+a}-1 .
\end{aligned}
$$

Akamine $^{4,53}$ made BASIC programs (type-1), in which the weighted least squared method is used and the optimization algorithm is Marquardt's method, to estimate the parameters of the standard formulae (20) combined with Eq. (32). We can obtain the estimated standard formulae (20) combined with Eq. (23) by using Akamine's BASIC programs and transformations (33) and (34). Kiso et al. ${ }^{\left({ }^{8}\right)}$ and Ikeda ${ }^{20)}$ used these programs which were modified to fix parameter $w_{\infty}$ because their data have few samples of large body sizes.

The most natural expansion of Eq. (22) may be

$$
\begin{aligned}
f(t)=1 & +A_{1} \cos 2 \pi\left(t-t_{1}\right)+A_{2} \cos 4 \pi\left(t-t_{2}\right)+\cdots \\
& +A_{n} \cos 2 n \pi\left(t-t_{n}\right) .
\end{aligned}
$$

Hewever, it is difficult to estimate parameters when the number of parameters $(2 n)$ increases. Therefore, Kiso et al. ${ }^{19)}$ used the following condition:

$$
t_{1}=t_{2}=\cdots=t_{n} .
$$

This condition was useful for their samples.

We can consider many conditions like this. For example,

$$
f(t)=1+A h(t)
$$

where

$$
\begin{aligned}
h(t)= & \cos 2 \pi\left(t-t_{1}\right)+\alpha_{1} \cos 4 \pi\left(t-t_{1}+\beta_{1}\right)+\cdots \\
& +\alpha_{n} \cos 2(n+1) \pi\left(t-t_{1}+\beta_{n}\right) .
\end{aligned}
$$


In this equation, the only parameters are $A$ and $t_{t}$, and the values of $\alpha_{i}$ and $\beta_{i}$ are decided by biological information (ex. spawning season, obstruction by high temperature).

For switched models, it is not so difficult to estimate parameters when $t_{2}$ and $t_{3}$ are constants decided by water temperature and biological information. However, it it difficult when they are parameters to be estimated. Pitcher and MacDonald"' used "fitting by hand" and "direct search" using a computer for parameter estimation with minnow data. However, there are few examples of this model because of the difficulty for parameter estimation and inferior suitability.

The switched sine function model is an example of a mathematical model which simulates the cumulative water temperature model. The original Pitcher and MacDonald model is switched by a cosine function which refers to water temperature. ${ }^{123}$ Therefore, this example model is regarded as a natural expansion of their model. However, this model is not easy for parameter estimation. In practice, it is better to estimate only $f^{*}(t)$ by the data of the cumulative water temperature first, and then to estimate the remaining growth parameters by the data of the growth.

The seasonal cessation model is an expansion of the switched model. However, this model does not correspond to the idea of the cumulative water temperature, and the amplitude $A$ is fixed at 1 . This model has many problems to be discussed.

\section{Acknowledgements}

I thank the late Mr. F. Kato of National Research Institute of Fisheries Science and Mr. K. Ishioka of Nansei National Fisheries Research Institute for kind help and advice, Dr. H. Matsuda of Kyushu University and Dr. K. Hiramatsu of National Research Institute of Far Seas Fisheries for helpful comments on the earlier version of this paper, and Dr. A. Hosomi, Dr. T. Hamano of Shimonoseki University of Fisheries and Dr. S. Miyai of Tohoku National Agricultural Experiment Station for important information. Comments from anonymous reviewers led to improvements in the manuscript.

\section{References}

1) F. J. Richards: A flexible growth function for empirical use J. Exp. Bot., 10, 290-300 (1959).
2) E. Ursin: On the incorporation of temperature in the von Bertalanfy growth equation. Medd. Danm. Fiskeri- og Havunders. N. S., 4, 1-16 (1963).

3) J. Moreau: Mathematical and biological expression of growth in fishes: Recent trends and further developements, in "Age and growth of fish" (ed. by R. C. Summerfelt and G. H. Hall), Iowa State University Press, 1987, pp. 81-113.

4) T. Akamine: Expansion of growth curves using a periodic function and BASIC programs by Marquardt's method. Bull. Jap. Sea Reg. Fish. Res, Lab., 36, 77-107 (1986).

5) T. Akamine: Estimation of parameters for Richards model. Bull. Jap. Sea Reg. Fish. Res. Lab., 38, 187-200 (1988).

6) Y. Hiyama and T. Akamine: Recent development of growth models in fisheries population dynamics (in Japanese). Toukeisuuri, 36, 104 (1988).

7) D. Pauly: The relationships between gill surface area and growth performance in fish: a generalization of von Bertalanffy's theory of growth. Meeresforsch, 28, 251-282 (1981).

8) J. Schnute: A versatile growth model with statistically stable parameters. Can. J. Fish. Aquat. Sci., 38, 1128-1140 (1981).

9) J. France and J. H. M. Thornley: Mathematical models in agriculture. Butterworths, London, 1984, pp. 75-94.

10) B.F. Theisen: Growth and mortality of culture mussels in the Danish Wadden Sea. Medd. Danm. Fiskeri- og Havunders. N. S., 6, 47 78 (1968).

11) A. Hosomi: Ecology of the mussel (in Japanese). Sankaidou, Tokyo, 1989, pp. 41-54.

12) T. J. Pitcher and P. D. M. MacDonald: Two models for seasonal growth in fishes. J. Appl. Ecol., 10, 599-606 (1973).

13) T. Akamine: Age analyses for size-frequency data (in Japanese). Bullet in of the Biometric Soc. of Japan, 8, 21-35 (1987).

14) T. Akamine: Estimation of age and growth based on length composition data (in Japanese), in "Numerical analysis for stock assessment" (ed. by Y. Shimadzu), Kouseisha Kouseikaku, Tokyo, 1987, pp. 118-131.

15) D. Pauly, M. Soriano-Bartz, J. Moreau, and A. Jarre-Teichmann: A new model accounting for seasonal cessation of growth in fishes. Aust. J. Mar. Freshwater Res., 43, 11511156 (1992).

16) R.S. Appeldoore: Modification of a seasonally oscillating growth function for use with mark-recapture data, J. Cons. int. Explor. Mer, 43, 194-198 (1987).

17) C. Pawlak and R. C. Hanumara: A comparison of nonlinear growth models for fisheries. Fish. Res, 11, 143-154 (1991).

18) I. F. Somers: On a seasonally oscillating growth function. Fishbyte, 6, 8-11 (1988).

19) K. Kiso, T. Akamine, S. Ohnishi, and Y. Matsumiya: Mathematical examinations of the growth of sea-run and fluviatile forms of female masu samon Oncorhynchus masou in River of the southern Sanriku district, Honshu, Japan. Nippon Suisan Gakkaishi, 58, 1779 -1784 (1992).

20) T. Ikeda: Growth and life history of the mesopelagic mysid Meterythrops microphthalma in the southern Japan Sea. Journal of Plankton Research, 14, $1767-1779$ (1992).

\section{Appendix A}

For the differential equation, the following initial condition is popular in mathematics:

$$
(t, w)=\left(0, w_{0}\right) \text {. }
$$

This condition and Eq. (15) give

$$
c=G\left(w_{0}\right)
$$

in general formula (16). Therefore, we obtain 
another particular solution to the Richards equation:

$$
w=\frac{w_{\mathrm{os}}}{\left[1+\left\{\left(w_{\infty} / w_{0}\right)^{r}-1\right\} \mathrm{e}^{-K t}\right]^{1 / r}} .
$$

This formula is popular in agricultural science.

For the expanded formula by using a periodic function, the initial condition (Al) gives

$$
c=G\left(w_{0}\right)-F(0),
$$

instead of Eq. (A2). Therefore, we obtain the following expanded formula:

$$
w=\frac{w_{\infty}}{\left[1+\left[\left(w_{\infty} / w_{0}\right)^{r}-1\right] \mathrm{e}^{-K(F(t)-F(0))}\right]^{1 / \tau}} .
$$

This formula is not easy for numerical calculation. It can be transformed to the standard formula $(20)$ by the following equation:

$$
F\left(t_{0}\right)=F(0)-G\left(w_{0}\right) .
$$

This equation is given by the initial conditions (21) and (A4).

\section{Appendix B}

Schnute simultaneous differential equations are written as

$$
\frac{\mathrm{d} Y}{\mathrm{~d} t}=Y Z
$$

$$
\frac{\mathrm{d} Z}{\mathrm{~d} t}=-Z(a+b Z)
$$

These equations give

$$
\mathrm{d} Z=-\frac{\mathrm{a}+b Z}{\mathrm{~d} Y}=
$$

This is a separation of variables type equation. Therefore, we obtain

$$
\frac{\mathrm{d} Z}{a+b Z}=\frac{\mathrm{d} Y}{Y} .
$$

Integrating both sides of this equation gives

$$
a+b Z=c Y^{-b},
$$

where $c$ is the integration constant. Substituting Eq. (B5) into Eq. (B1) gives

$$
\mathrm{d} Y=Y^{c Y^{-b}-a}-a Y^{1-(c / a) Y^{-b}}
$$

This is the Richards differential equation shown by the following transformation:

$$
Y \rightarrow w, a \rightarrow K, b \rightarrow-r, c \rightarrow \frac{K}{w_{\infty}{ }^{r}} .
$$

We can also derive Eqs. (B1) and (B2) from Eq. (B6) by the backward way. Therefore, Schnute simultaneous differential equations are identical to the Richards differential equation. 